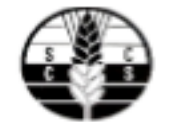

\title{
RESPUESTA DEL TOMATE (Lycopersicon esculentum Mill) A UNA ENMIENDA CALCICA EN UN SUELO ÁCIDO DE SUCRE - COSTA CARIBE COLOMBIANA
}

\author{
Luis Fernando Acosta Perez ${ }^{1} \bowtie$, y Eliecer Cabrales Herrera ${ }^{2}$.
}

1 Ingeniero Agrícola, MSc., Grupo de Investigación Agricultura sostenible, Universidad de Córdoba. ORCID 0000-0003-3111-

6121.

aclufe@hotmail.com

2 Ingeniero Agrónomo, $P h D$. Profesor área suelos, Grupo de Investigación Agricultura sostenible, Universidad de Córdoba. ORCID 0000-0002 8409-677X.

PALABRAS CLAVES: Acidez, calcio, fruto, rendimiento, producción.

\section{RESUMEN}

En aras de contribuir a la seguridad alimentaria y a la motivación de los pequeños productores de hortalizas del departamento de Sucre (Colombia), se implementó este ensayo bajo la premisa de que el calcio en el suelo contribuye a tener plantas con pared celular mucho más resistentes al ataque fúngico y con ello se disminuye la aparición de enfermedades. Con base en lo anterior, se estableció este ensayo en cultivo de tomate, en la que se establecieron cinco (5) dosis de calcio $(0,1,2,3$ y 4 ton.ha1) y tres repeticiones en un diseño completamente al azar. Como fuente de calcio se utilizó un producto comercial que contiene $37 \%$ de $\mathrm{CaO}$ y 5,3\% de MgO. El objetivo fue evaluar el efecto de diferentes dosis de calcio en los componentes del rendimiento del cultivo del tomate Chonto en condiciones semicontroladas en Sucre - Caribe colombiano. El suelo fue de reacción ácida y de baja oferta nutricional. Se utilizó un diseño completamente al azar con tres repeticiones. La información fue almacenada en tablas Excel y procesada con el programa estadístico SAS 9.0, se hizo análisis de varianza y de regresión. Se encontró que las dosis 2 y 3 ton.ha- 1 favoreció el crecimiento de las plantas de tomate, aumentando dimensiones del fruto, peso del fruto y rendimiento y los modelos que explican esas tendencias son de orden lineal.

\section{TOMATO RESPONSE (Lycopersicon esculentum Mill) TO A CALCIUM AMENDMENT IN A SUCRE ACID FLOOR - COLOMBIAN CARIBBEAN COAST}

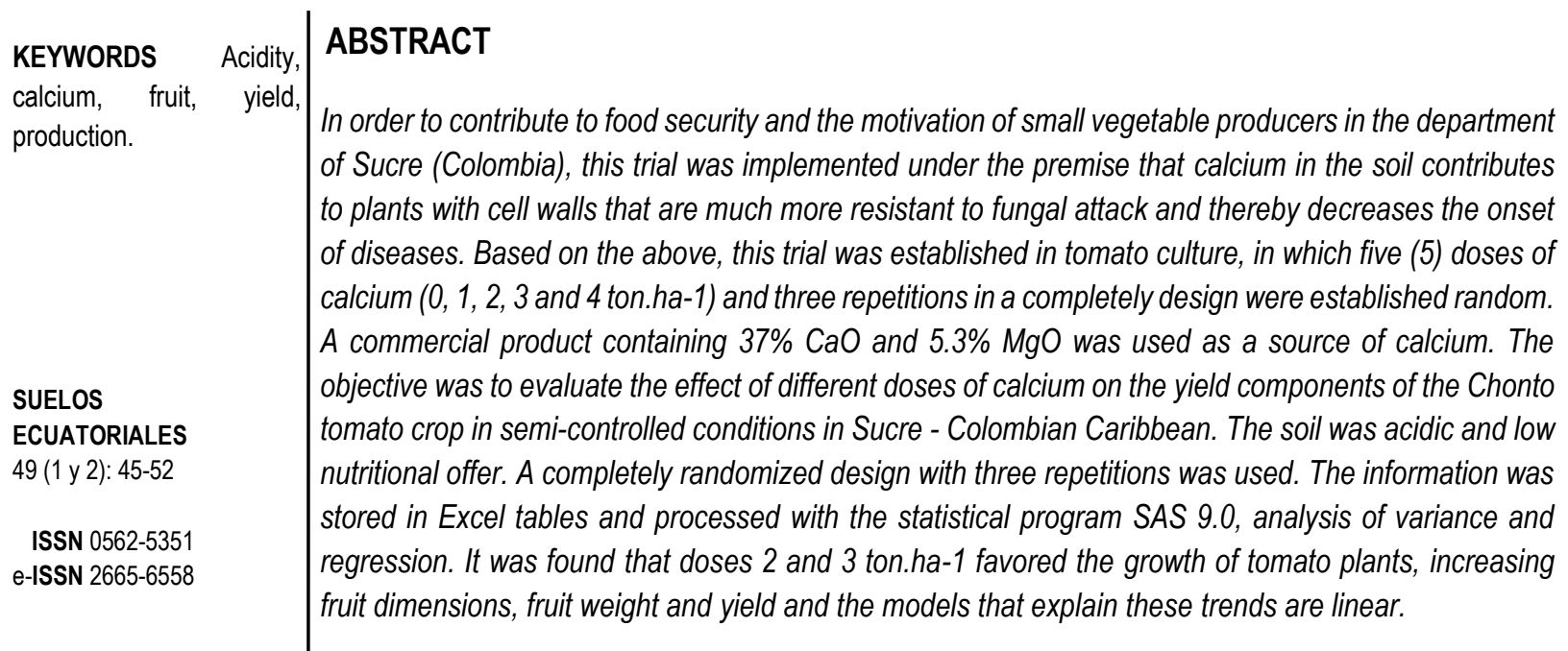

Rec.: 11.11.2019

Acep.02.12.2019 


\section{INTRODUCCIÓN}

Aproximadamente el $40 \%$ de los suelos cultivables del mundo son ácidos y potencialmente tienen efectos de toxicidad por aluminio, hierro y manganeso, lo cual suele suceder cuando el pH es inferior a 5.5, bajo estas condiciones, el aluminio aparece en la solución del suelo como $\mathrm{Al} 3+$, que es un ion libre que puede formar compuestos con otros iones presentes en la solución como los fosfatos y los sulfatos. Estos iones de Al3+ también pueden competir con otros cationes como $\mathrm{Ca} 2+$ y Mg2+ por los sitios de intercambio en los coloides del suelo; de esta forma el aluminio intercambiable puede ser un factor limitante de la fertilidad de la mayoría de los suelos ácidos (Zapata, 2004).

La deficiencia de calcio muchas veces se asocia al grado de acidez que pueda tener el suelo, y rara vez a la no absorción y desajustes metabólicos que se dan en las plantas, por lo que es necesario evaluar su disponibilidad o aprovechabilidad por las mismas (García, 2000; Castellanos et al., 2003). El calcio es de lenta movilidad, tanto en el suelo como en la planta, lo cual obliga a replantear las técnicas de fertilización con este elemento, es decir, en lo posible debe estar en el suelo en forma soluble, suficiente y disponible para la planta (Li et al., 2001). Aunque estudios recientes han demostrado que puede entrar en pequeñas cantidades vía apoplasto (por las hojas y frutos), cantidad que resulta insuficiente para satisfacer las necesidades de las plantas (Dayod et al., 2010), es decir, que la fertilización foliar con este elemento no satisface en su totalidad los requerimientos de las plantas (Lazcano 2000, Rodríguez et al. 2002, Taylor et al. 2004).

El tomate es una planta que demanda altas cantidades de nutrientes y la producción de frutos de buen calibre y alto peso está relacionada con la disponibilidad de nutrientes en cada una de las etapas de crecimiento del cultivo (Vallejo \& Estrada, 2004). Para el cultivo de tomate se requieren suelos sueltos, bien drenados, profundos y ricos en materia orgánica (Espinosa, 2004; Jaramillo et al., 2006).
Según Zapata (2004), el rango de pH adecuado para el cultivo de tomate está entre 5,5 y 6,5.

Por lo anterior, se estableció el ensayo en la que se evaluaron diferentes dosis de calcio aplicado al suelo, utilizando como fuente un producto comercial que contiene $37 \%$ de $\mathrm{CaO}$, en la que evaluaron parámetros del rendimiento del cultivo del tomate en condiciones semicontroladas en Sucre - caribe colombiano.

\section{MATERIALES Y METODOS}

Ubicación. La investigación se realizó en condiciones semicontroladas en Sincelejo - Sucre (Caribe colombiano), altura de $213 \mathrm{msnm}$, con temperatura promedia de $27^{\circ} \mathrm{C}$, humedad relativa promedia de $80 \%$, y precipitación promedia anual de $1164 \mathrm{~mm}$.

Selección y preparación del sustrato. El muestreo de suelos se hizo en la finca Las Lauras del corregimiento Santa Clara, municipio El Roble del departamento de Sucre, suelo de reacción ácida. El suelo para el llenado de las bolsas se tomó de los primeros $20 \mathrm{~cm}$ de profundidad, el cual se secó a sombra, se tamizó a $4 \mathrm{~mm}$, y se homogenizó, para posteriormente hacer el llenado de las bolsas con capacidad de $20 \mathrm{~kg}$. Posteriormente las bolsas se ubicaron en hileras separadas a $80 \mathrm{~cm}$ y en las hileras se ubicarán a cada $60 \mathrm{~cm}$.

Toma de datos y diseño. Se utilizó un diseño completamente al azar (DCA) con cinco tratamientos y 3 repeticiones, cada unidad experimental estuvo conformada por 5 bolsas. Los tratamientos fueron: $0,1,2,3$ y 4 ton.ha-1 de producto comercial con $37 \%$ de $\mathrm{CaO}$. La información obtenida se colectó tablas Excel y se procesó mediante el programa estadístico SAS versión 9.0, se hizo el análisis de varianza y regresión. Se aplicó la prueba de comparación de medias de Tukey con un nivel del $95 \%$ de confianza. Manejo agronómico del ensayo. Se hizo en forma tradicional, las arvenses se manejaron manualmente. De las plagas, la única de interés que se presentó fue el minador de follaje, se controló con el producto comercial Hibertrina, con éxito en el control. Se utilizó un plan de fertilización 100-80-100 de N-P-K, utilizando como fuentes la Urea, DAP y 
$\mathrm{KCl}$ para N, P y K, respectivamente. Los elementos menores se aplicaron en forma foliar, para lo cual se aplicaron cada 10 días aspersiones de $5 \mathrm{ml}$ de producto comercial por litro de agua. Se utilizó el producto comercial WUXAL.

Parámetros evaluados. Se midieron los componentes del rendimiento: número de frutos cosechados, peso del fruto, dimensiones del fruto (longitud y diámetro) y rendimiento.

\section{RESULTADO Y DISCUSIÓN}

Número de frutos cosechados. El análisis de varianza arrojó que existe diferencia altamente significativa entre los tratamientos (Tabla 1), mediante una prueba de comparación de medias de Tukey $(0,05)$, se corrobora que el tratamiento testigo $\mathrm{T} 0$, es diferente a los tratamientos T2, T3 y T4, pero similar al tratamiento T1 (Figura 1), indicando que el calcio influye positivamente en el número de frutos cosechados. Esta figura muestra una tendencia positiva que puede ser explicada en un $78 \%$ por un modelo de orden lineal, dicho modelo indica que hay relación directa entre las dos variables, lo que evidencia que la relación entre el número de frutos cosechados y la aplicación de las dosis de calcio, es una relación fuerte. Esto se debe posiblemente a que el calcio se unió con los grupos de las pectinas y se produjo el pectato de calcio, quien le da resistencia a la pared celular contra el ataque de los patógenos al fruto (Cabrales, 2010), por lo que se disminuye la probabilidad de caída de frutos y aparición de enfermedades tipo fúngico. En este sentido Lazcano (2000), indica que cuando hay disponibilidad de calcio en el suelo, se incrementa la producción de sustancias en las plantas que dificultan la penetración de patógenos al fruto.

Tabla 1. Análisis de varianza para las variables respuestas evaluadas

\begin{tabular}{llccccc}
\hline & F.V & & \multicolumn{3}{c}{ Análisis de Varianza } & \\
& & GL & SC & CM & F & p-valor \\
\hline Frutos & Dosis de & 4 & $1.323,07$ & 330,77 & 34,94 & $<0,0001$ \\
cosechados & Ca & & & & & \\
& Error & 10 & 94,67 & 9,47 & & \\
& Total & 14 & $1.417,74$ & & & \\
Peso de fruto & Dosis de & 4 & 518,93 & 129,73 & 94,74 & $<0,0001$ \\
& Ca & & & & & \\
& Error & 10 & 13,69 & 1,37 & & \\
Longitud fruto & Total & 14 & 532,62 & & & \\
& Dosis de & 4 & 66,17 & 16,54 & 9,19 & \\
& Ca & & & & & \\
& Error & 10 & 18,00 & 1,80 & & \\
Diámetro fruto & Total & 14 & 84,17 & & & \\
& Dosis de & 4 & 47,76 & 11,94 & 51,39 & $<0,0001$ \\
& Ca & & & & & \\
& Error & 10 & 2,32 & 0,23 & & \\
& Total & 14 & 50,08 & & & \\
Rendimiento & Dosis de & 4 & $3.096,74$ & 774,19 & 66,59 & $<0,0001$ \\
& Ca & & & & & \\
& Error & 10 & 116,25 & 11,63 & & \\
& Total & 14 & $3.212,99$ & & & \\
\hline
\end{tabular}

Por otra parte posiblemente no hubo rotura enzimática de las protopectinas que componen el armazón de la pared celular, la cual no fue degrada por agentes oxidantes, y de esta forma dichas paredes no se reticulan, las células no pierden compartimentación y el fruto no pierde firmeza (Dhall 
\& Singh, 2013; Saure, 2014; Kiferle et al., 2013; Landini, Gonzali, \& Perata, 2011), dificultando así el ataque de patógenos al fruto. Los resultados de esta investigación difieren a los reportados por Zamban et al. (2018); Arruda Júnior et al. (2011); Rab y Haq (2012) y Cabrales (2010).

Peso de fruto. Según la Tabla 1, el análisis de varianza arrojó que hay diferencias altamente significativas $(p<0.01)$ entre los tratamientos. Mediante la prueba de comparación de medias de Tukey $(0,05)$, se comprobó que el mayor peso en frutos se logró en los tratamientos T2, T3 y T4 que a su vez son estadísticamente similares, pero difieren de los tratamientos T0 y T1 donde se presentaron los valores de peso de fruto más bajos (Figura 2). A medida que se adiciona calcio al suelo, el peso del fruto tiende a incrementarse, como se puede apreciar en la figura 2, donde se muestra la tendencia que puede ser explicada en un $82,56 \%$ por un modelo de orden lineal, dicho modelo indica que hay relación entre las dos variables, lo que evidencia que la relación entre el peso de frutos y la aplicación de las dosis de calcio al suelo, es una relación fuerte. Este hallazgo posiblemente se debe a que, al existir más calcio disponible, éste puede ser absorbido e incorporado en las partes celulares e influir en la división de las células para inducir el crecimiento y peso de los frutos. Además, pudo ocurrir que las cantidades de calcio que no lograron entrar al citosol quedan en la pared celular, situación que genera células más pesadas, como lo indica Marschner (2002). Cuando el calcio se suministra adecuadamente, proporciona mayor establecimiento de fruta, mayor crecimiento y menor incidencia de trastornos fisiológicos, junto con un creciente número y peso de frutos de tomate (Zamban et al. 2018).

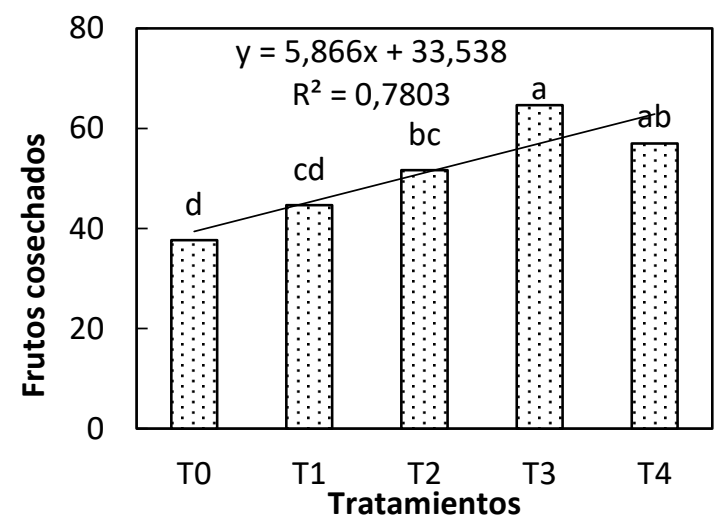

Figura 1. Frutos cosechados del cultivo de tomate en función a la cantidad de calcio aplicado al suelo. Letras iguales no difieren estadísticamente según la prueba de Tukey $(p=0,05)$

Estos resultados son coherentes con los reportados por Zamban et al. (2018) y Martinez-Damián et al., 2019, quienes obtuvieron mayor peso del fruto de tomate, a medida que se aumentaba la dosis de calcio. Pero difieren a los encontrados por Arruda Júnior et al. (2011) quienes verificaron que, a medida que aumenta la dosis de calcio, se observa una disminución en el peso medio de las frutas. También difieren a los reportados por Cabrales

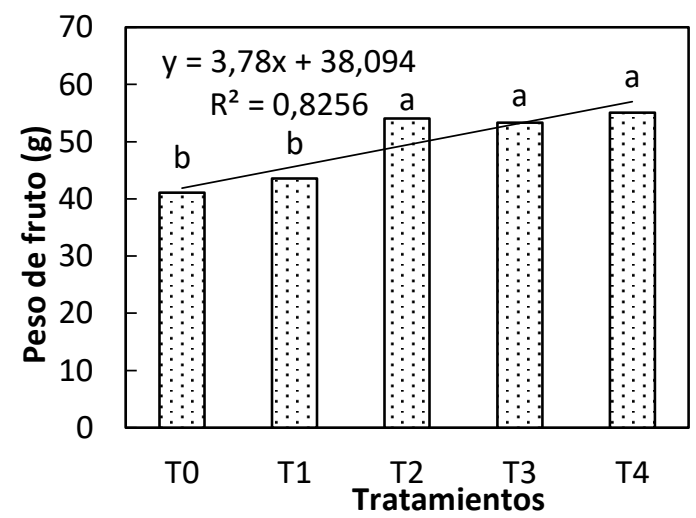

Figura 2. Peso del fruto del cultivo de tomate en función a la cantidad de calcio aplicado al suelo. Letras iguales no difieren estadísticamente según la prueba de Tukey $(p=0,05)$

(2010) quien evaluó la respuesta a la aplicación de calcio sobre la pudrición apical del fruto del tomate en el Valle Medio del Sinú, donde obtuvo el máximo peso de frutos de $124 \mathrm{~g}$. De igual manera, también están por debajo de $129 \mathrm{~g}$ que reporta Muhammad et al., (2012) y de los 96,4 g que reporta Martínez et al. (2013). 
Dimensiones de fruto. El análisis de varianza indica que hay diferencias altamente significativas para los tratamientos (Tabla 1), tanto en longitud como en diámetro del fruto. Con la prueba de comparación de medias de Tukey (0.05), se constató que el tratamiento testigo T0 es similar estadísticamente a los tratamientos T1, T3 y T4, pero diferente al tratamiento T2 y a la vez el tratamiento $\mathrm{T} 2$ es estadísticamente igual al tratamiento T1. La longitud de fruto presenta una tendencia ascendente hasta la dosis de 2 ton/ha, de allí en adelante desciende la longitud del fruto (Figura 3), indicando que dosis muy bajas y altas no favorecen la longitud del fruto de tomate en esta investigación. Esto posiblemente se deba a que la dosis de 2 ton/ha fue la que produjo el equilibrio que pudo brindar la aplicación de calcio al suelo, lo que a su vez pudo influir positivamente para que la planta lo tomara junto con otros nutrientes e incrementara su tolerancia al ataque de patógenos al fruto, como lo afirma Lazcano (2000) y Espinosa (2000). A demás, lo anterior puede sugerir que la aplicación de la dosis de 2 ton/ha, puede estar involucrada de forma positiva en la división y elongación celular de los frutos recién cuajados, favoreciendo un mayor tamaño, tal como lo señala Uchanski y Blalock (2013).

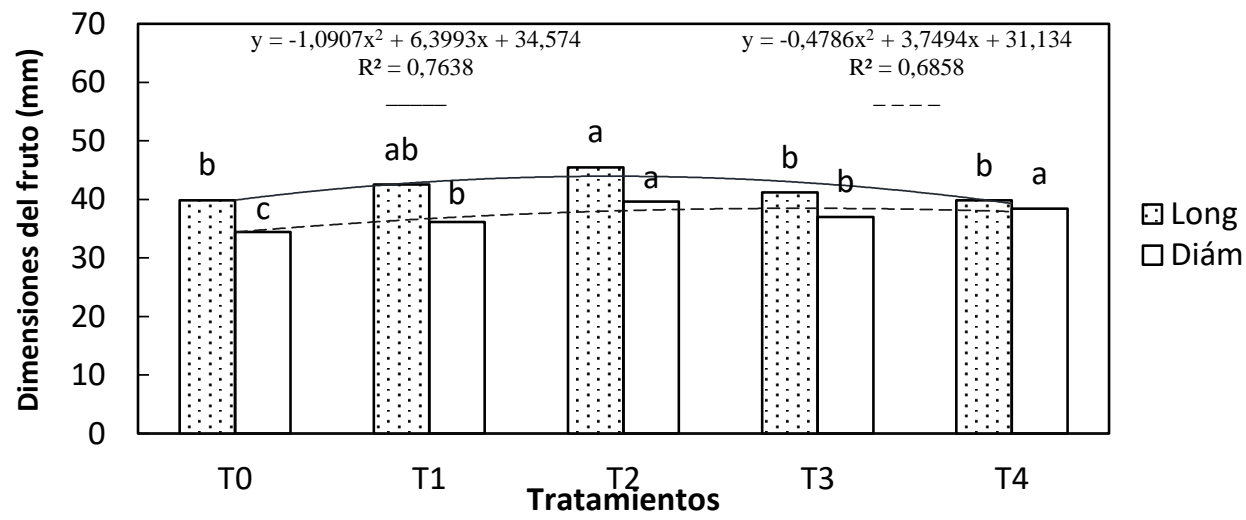

Figura 3. Dimensiones del fruto del cultivo de tomate en función a la cantidad de calcio aplicada al suelo. Letras iguales no difieren estadísticamente según la prueba de Tukey $(p=0,05)$

Según la figura 3 el tratamiento testigo T0 es diferente a al resto de los tratamientos, a medida que se adiciona calcio al suelo, el diámetro de fruto tiende a aumentar hasta la dosis de 2 ton/ha, con las dosis de 3 y 4 ton/ha tiende a mantenerse constante el diámetro del fruto, dejando entrever que dosis superiores a 2 ton/ha suelen ser inaprovechables para dicha planta. El incremento en el diámetro del fruto de tomate se debe posiblemente, a que el calcio suministrado al suelo haya influido en la extensibilidad epidermal (Thompson, 2001) y en el desarrollo de los tejidos del pericarpio, los cuales, generalmente comprenden más de las $2 / 3$ partes del total del peso del fruto, esta división y elongación de los tejidos del pericarpio son determinantes para el crecimiento del fruto de tomate. A demás posiblemente el aumento en el diámetro del fruto, esté asociado con el incremento en el número y tamaño de las células como lo indica MartinezDamián et al., 2019 y Shinozaki et al., 2015. Los resultados de esta investigación son similares a los reportados por Martinez-Damián et al., 2019; Zamban et al. (2018); Silva et al., (2009), pero difieren a los reportados por Afsana et al., (2017), Abbasi et al., (2013), Rab y Haq (2012) y Cabrales (2010).

Rendimiento. El análisis de varianza arrojó diferencias altamente significativas para los tratamientos (tabla 1). El tratamiento testigo T0 y el T1 (1 ton/ha de calcio) son similares estadísticamente, pero diferentes a los demás tratamientos (figura 4). La respuesta de la variable rendimiento en función a la cantidad de cal aplicada 
al suelo, se describe en la figura 4, donde se observa la tendencia que puede ser explicada en un $85,23 \%$ por un modelo de orden lineal, dicho modelo indica que hay relación directa entre las dos variables, lo que evidencia que la relación entre rendimiento y la aplicación de las dosis de calcio, es una relación fuerte. Posiblemente se deba a que la disponibilidad de calcio en el suelo mejoró la reacción del suelo y haya contribuido a mejorar el equilibrio químico del mismo, cuando esto ocurre, las plantas se nutren mucho mejor $y$, por ende, serán más tolerantes al ataque de patógenos. Pudo ocurrir que no se afectaron adversamente los procesos fisiológicos de la planta, y probablemente resultaron en una cantidad mayor de fotoasimilados, este probable aumento de fotoasimilados en la planta proporcionó una mayor cantidad de frutos, aumento del tamaño del fruto y mejora en la calidad del fruto, aumentando el rendimiento, así como lo indica Zamban et al. (2018); Caliman, (2003). En contraste con nuestros resultados, Afsana et al., (2017), Kazemi (2013) y Lolaei (2012), reportaron que la aplicación de calcio en presencia de ácido salićlico aumentó el rendimiento del tomate, con producción promedio de 72.57 ton/ha. A demás estos resultados son coherentes con los reportados por Zamban et al. (2018), pero diferentes a los Arruda Júnior et al. (2011); Rab y Haq (2012); Cano et al. (2011); Cabrales (2010) y Machado et al. (2007).

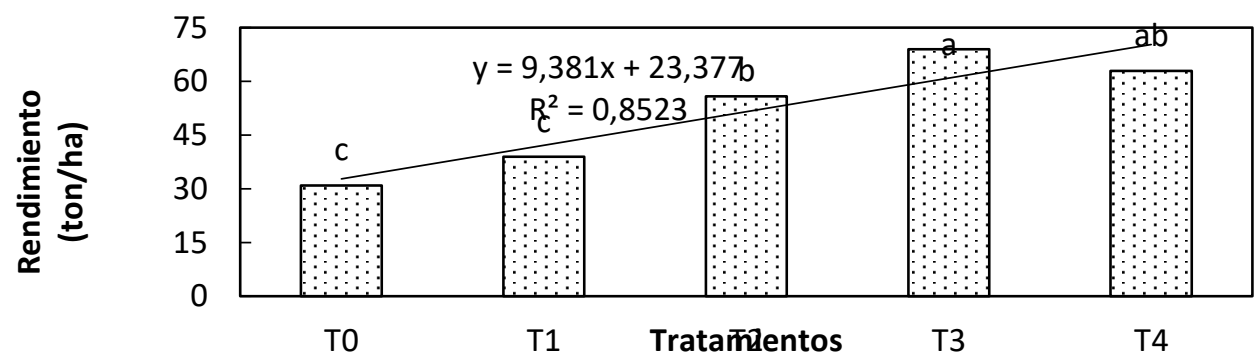

Figura 4. Rendimiento del cultivo de tomate en función a la cantidad de calcio aplicado al suelo. Letras iguales no difieren estadísticamente según la prueba de Tukey $(\mathrm{p}=0,05)$

\section{CONCLUSIONES}

La aplicación de calcio al suelo de baja oferta nutricional como los suelos ácidos de Sucre, mejora los componentes del rendimiento del tomate Chonto. La mejor dosis de calcio aplicado al suelo para el cultivo de tomate Chonto en suelos de baja oferta nutricional de Sucre fue la de 3 t/ha.

\section{AGRADECIMIENTOS}

Los autores agradecen el apoyo administrativo, técnico y económico otorgado por la Gobernación de Sucre, al Departamento Administrativo de Ciencia, Tecnología e Innovación (Colciencias), a la Universidad de Córdoba y a la Corporación Universitaria del Caribe (CECAR).

\section{REFERENCIAS}

Abbasi, N., Zafar, L., Khan, H., Qureshi, A. 2013. Effects of naphthalene acetic acid and calcium chloride application on nutrient uptake, growth, yield and post- harvest performance of tomato fruit. Pak. J. Bot:,45(5): 1581-1587.

Afsana, N., Mahbub, M., Hossain, E., Nizam, R., Monalesa, N., Hussain, A., Parvin, S. 2017. Response of Tomato (Solanum lycopersicum L.) to Salicylic Acid and Calcium. Journal of Applied Life Sciences International. 15(2): 1-7.

Arruda Júnior, S. J.; Bezerra Neto, E.; Barreto, L. P.; Resende, L. V. 2011. Podridão apical e 
produtividade do tomateiro em função dos teores de cálcio e amônio. Revista Caatinga, v.24, p.20-26.

Cabrales, E. 2010. Respuesta a la aplicación de calcio sobre la pudrición apical del fruto del tomate en el Valle medio del Sinú (Córdoba, Colombia). Suelos Ecuatoriales, 40(1): 68-72.

Caliman, F.R.B. 2003. Produção e qualidade de frutos de genótipos de tomateiro em ambiente protegido e no campo. Tese de mestrado. Universidade Federal de Viçosa, Viçosa-MG, Brazil.

Cano, S., Gallego, M., y Chavarriaga, W. 2011. Efecto de la aplicación de calcio y fósforo en un suelo ácido y la respuesta en el cultivo de tomate chonto (Solanum lycopersicum (L.) Mill). Agron. 19(1): $77-87$.

Castellanos, G., Quijada, O., Guanipa, N., Camacho, R., Fonseca, Y. 2003. Control de la pudrición apical del fruto y secamiento del árbol mediante manejo integral del cultivo de guayaba (Psidium guajava L.). Bioagro 15(2): 135-142.

Dayod, M., Donald, S., Allen, R., Gilliham, M. 2010. Calcium storage in plants and the implications for calcium biofortification. Protoplasma. Australia: Springer. 42p.

Dhall, R. K., \& Singh, P. (2013). Effect of ethephon and ethylene gas on ripening and quality of tomato (Solanum Lycopersicum L.) during cold storage. Journal of Nutrition \& Food Sciences, 3(6), 244. doi: 10.4172/2155- 9600.1000244

Espinosa, J. 2000. Manejo de nutrientes en agricultura por sitio específico en cultivos tropicales. Informaciones agronómicas de INPOFOS, 39: 2-6.

Espinosa-Zapata, C. 2004. Producción de tomate en invernadero. Invernaderos: Diseño, manejo y producción. pp. 19-43. Memorias del Simposio Nacional de Horticultura. Torreón, México.
García, J. 2000. Importancia del calcio en la nutrición de las plantas. En: $X$ congreso Nacional de la Ciencia del Suelo, resúmenes.

Jaramillo, J., Rodríguez, V., Guzmán, M. \& Zapata, M. 2006. El cultivo de tomate bajo invernadero. Boletín Técnico 21. Corpoica La Selva. Rionegro, Antioquia, Colombia.

Kazemi, M. 2013. Foliar application of salicylic acid and calcium on yield, yield component and chemical properties of strawberry. Bull. Env. Pharmacol. Life Sci, 2(11): 19-23.

Kiferle, C., Gonzali, S., Holwerda, T. H., RealIbaceta, R., \& Perata, P. (2013). Tomato fruits: a good target for iodine biofortification. Frontier in Plant Science, 4, 205. doi: 10.3389/fpls.2013.00205 Landini, M., Gonzali, S., \& Perata, P. (2011). lodine biofortification in tomato. Journal of Plant Nutrition and Soil Science, 174(3), 480-486. doi: 10.1002/jpln.201000395

Lazcano, I. 2000. Deficiencia de Ca en tomate (Lycopersicon esculentum Mill). Informaciones Agronómicas, Instituto de la Potasa y el Fósforo de Canadá, 39: 7-8.

Li, Y., Stanghellini, C., Challa, H. 2001. Effect of electrical conductivity and transpiration on production of greenhouse tomato (Lycopersicon esculentum L). Scientia Horticulturae 88: 1129 1135.

Machado, A.Q., M.A.R. Alvarenga, and C.E.T. Florentino. 2007. Produção de tomate italiano (saladete) sob diferentes densidades de plantio e sistemas de poda visando ao consumo in natura. Hortic. Bras. 25, 149-153. Doi: 10.1590/S010205362007000200004

Marschner, H. 2002. Mineral nutrition of higher plants. 2nd ed. Academic Press London. 889p.

Martínez, L., Velasco, V., Ruiz, J., Enríquez, J., Campos, G., y Montaño, M. 2013. Efecto del nitrato de calcio y sustratos en el rendimiento del tomate. 
Revista Mexicana de Ciencias Agrícolas publicación especial número 6: 1175-1184.

Martínez-Damián M. T., Cano-Hernández, R., Moreno-Pérez, E. C., Sánchez-del Castillo, F., \&, Cruz-Álvarez, O., (2019). Effect of preharvest growth bioregulators on physicochemical quality of saladette tomato. Revista Chapingo Serie Horticultura, 25(1), 29-43. doi: 10.5154/r.rchsh.2018.06.013.

Muhammad, A., Wong, C., y Mahamud, S. 2012. The efficacy of calcium formulation for treatment of tomato blossom-end rot. J. Trop. Agric. and Fd. Sc. 40(1): 89-98.

Rab, A. y Haq I. 2012. Foliar application of calcium chloride and borax influences plant growth, yield, and quality of tomato (Lycopersicon esculentum Mill.) fruit. Turk J Agric. 36: 695-701.

Rodríguez, D., Pontes, A., Minami, K., Dos Santos, D. 2002. Quantidade absorvida e concentrações de micronutrientes em tomateiro sob cultivo protegido. Sci. Agric 59(1): 137-144.

Saure, M. C. (2014). Why calcium deficiency is not the cause of blossom-end rot in tomato and pepper fruit - a reappraisal. Scientia Horticulturae, 74, 151154. doi: 10.1016/j.scienta.2014.05.020

Shinozaki, Y., Hao, S., Kojima, M., Sakakibara, H., Ozeki-lida, Y., Zheng, Y., Fei, Z., Zhong, S., Giovannoni, J. J., Rose, J. K., Okabe, Y., Heta, Y., Ezura, H., \& Ariizumi, T. (2015). Ethylene suppresses tomato (Solanum lycopersicum) fruit set through modification of gibberellin metabolism. The Plant Journal, 83(2), 237-251. doi: 10.1111/tpj.12882

Silva, M.W., C.J. Jadoski, E.O. Ono, and R. Goto. 2009. Cálcio, boro e reguladores vegetais na fixação de frutos em tomateiro. Pesqu. Aplicada Agrotecnol. 2(3), 103-112.
Taylor, M. y Locascio, S. 2004. Blossom-End Rot: A Calcium Deficiency. Journal of Plant Nutrition 27(1): 123-139.

Thompson, D.S. 2001. Extensiometric determination of the rheological properties of the epidermis of growing tomato fruit. Journal of Experimental Botany 52: 1291-1301.

Uchanski, M. E., \& Blalock, A. (2013). Ethephon improved pigmentation but had no effect on cayenne pepper fruit yield in southern New Mexico. Hortscience, 48(6), 738-741. Retrieved from http://hortsci.ashspublications.org/content/48/6/738. full.pdf+html

Vallejo C., F.A. \& Estrada S., E.I. (2004). Producción de hortalizas de clima cálido. Palmira. Universidad Nacional de Colombia.

Zamban, D. T., Prochnow, D., Caron, B. O., Turchetto, M., Fontana, D. C., \& Schmidt, D. (2018). Aplicaciones de calcio y boro aumentan la producción de híbridos de tomate Italiano (Solanum lycopersicum) en dos temporadas de cultivo. Revista Colombiana De Ciencias Hortícolas, 12(1), 8293. doi.org/10.17584/rcch.2018v12i1.7338

Zapata-Hernández, R.D. (2004). La química de la acidez del suelo. Medellín. Universidad Nacional de Colombia. 